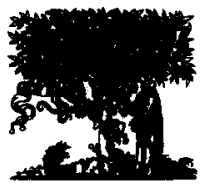

ELSEVIER

European Journal of Operational Research 91 (1996) 211-213

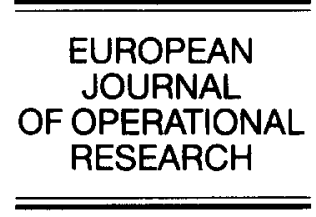

\title{
Editorial
}

\section{The virtual reality of financial-economic decision making}

\author{
Erio Castagnoli ${ }^{\text {a }}$, Jaap Spronk ${ }^{\text {b }}$ \\ ${ }^{a}$ Bocconi University, Milano, Italy \\ ${ }^{\mathrm{b}}$ Erasmus University, Rotterdam, The Netherlands
}

The world in which we are living (our world) becomes progressively more symbolic and abstract (than in the past, than the simple natural world of our fathers). Television and advertising images are a sort of new reality; teleconferences are gradually replacing personal (direct) communication; computers are increasingly conditioning our lives and habits; wild nature is symbolized by well organized national parks: virtual reality may finally take the place of physical reality.

The economy and economic science are not an exception to this line of 'progress'. The real economy is losing ground to the financial economic system which, in turn, creates more and more sophisticated and immaterial instruments. We may or may not like this process but in order to navigate properly, we have to get as much insight as possible into the rules of our (brave new) world with its increasing complexity and abstraction. Furthermore, we have to develop frameworks and tools which help to make better decisions. Financial and non-financial institutions in Europe have to face two problems: to be competitive in the extremely efficient world markets and to cope with local circumstances created by many very different local markets and institutional arrangements. The objective of the Euro Working Group on Financial modelling is to emphasize both problems, but mainly the second one, till recently disregarded by the standard financial literature. We would be very proud in thinking that a small cause of the interest in this class of problems is somehow connected with our constancy in giving them the same citizenship rights as standard models in financial-economic literature.

Ten years ago the EURO Working Group on Financial Modelling was founded: it was an attempt to build a European and decision-oriented approach to the study of financial-economic problems. An approach which tries to integrate the attainments of financial-economic theory with those of disciplines as operational research and management science, taking account of the peculiarities of the different (financial-) economic systems.

Our group is formed by more than four hundred people from over thirty countries from all continents but mainly European. They have fairly different backgrounds: the majority is represented by academics coming from a large variety of fields such as financial economics, business science, statistics, applied mathematics, management science, operational research and banking. Some of these have a considerable experience with financial-economic decision making in practice. The same holds of course even more for the small but stable group of practitioners attending the meetings. This 'melting pot' is in our opinion the ideal environment for people to meet and to exchange results and - last but not least - to develop new ideas. For this reason we kept the sustained pace of two meetings every year: partly devoted to the presentation and discussion of the results of completed research, partly to discuss projects and new lines of research; always within a co-operative and constructive setting.

The working group has produced a series of 
proceedings, special issues and working papers. More information on the group and its publications can be obtained from the secretary of the group,

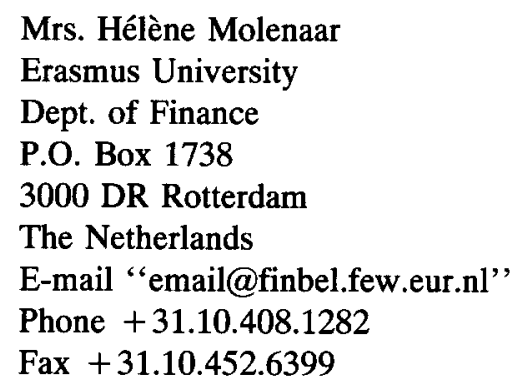

Over the years, the European Journal of Operational Research has published many papers in the realm of financial modelling, both in special issues and in regular issues. The present special issue is the first being published in EJOR in co-operation with the EURO Working Group on Financial Modelling. The working group will continue to co-operate with the editors of EJOR by producing a regular stream of so-called feature issues devoted to financial modelling.

An announcement and a call for papers for these feature issues can be found elsewhere in the present issue.

The invitation to submit to the present special issue went to all the members of the working group. Furthermore, it was distributed at various conferences. All papers received were refereed by several referees. The acceptance rate turned out to be about $30 \%$. The resulting special issue offers an interesting portfolio of high quality contributions, which we hope will inspire to submit to the future feature issues.

The first paper, by Niklas Ekvall, presents a lattice approach for pricing of multivariate contingent claims. The approach avoids the possibility of negative jump probabilities and possesses an attractively fast rate of convergence. L. Peter Jennergren and Bertil Näslund deal with the pricing of a class of options that have a fixed date of maturity but may be cancelled prematurely. The main result is an extension of the Black-Scholes formula to options where the time to expiration is stochastic. Anna Rita Bacinello and Fulvio Ortu propose a pricing model for life insurance policies in which the benefits are linked to the performance of a portfolio of interest rate sensitive assets (reference fund) and in which a minimum guarantee provision is made.

Anton Pelser and Ton Vorst demonstrate that, when transaction costs are taken into account, efficient dynamic portfolio strategies are more costly than simple and popular strategies such as stop-loss and lock-in. Furthermore, they show that the use of options reduces the initial costs of both simple and efficient strategies. Christiana Vassiadou-Zeniou and Stavros Zenios integrate traditional simulation models for bond pricing with recent developments in robust optimization to develop tools for the management of portfolios of callable bonds. Two models are developed and back-tested, performing consistently well. Thomas Wiesemann discusses a consumption plan for the intertemporal portfolio and consumption problem in which in each period a proportion of the portfolio value in time zero is consumed that equals the risk-adjusted portfolio return in that period. He shows that the portfolio value of this plan can be preserved over time.

Emilio Barucci and Leonardo Landi study a linear model for a future market characterized by the presence of different classes of traders. They analyze how the fact there are different classes of traders affects rational expectations equilibria of the model with respect to bounded rationality learning. Marisa Cenci, Annamaria Cerquetti and Lorenzo Peccati study the phenomenon of imitation in stock markets. They show that in a number of settings, imitation can generate instability in stock market equilibria. Andrew Pollock and Mary Wilkie present a framework for examining the quality of currency forecasts. Their framework gives components of accuracy that are appropriate for statistically derived and judgementally based forecasts. The framework is applied to forecasts from three major banks.

Lenos Trigeorgis deals with the numerical evaluation of leasing contracts with a variety of embedded operating options. He describes and illustrates a numerical analysis method for valuing leasing contracts that is based on a Contingent Claims Analysis. Claudia Battaglio, Giovanni Longo and Lorenzo Peccati address the structure of fees in a consumer credit contract. They show how, by using a linear programming approach, the fee structures can be optimized while taking account of the legal profile of the 
contract. Robert C. Coppes and Evert Jan Stokking develop a method of credit risk exposure in which the exposure is a function of interest rates, exchange rates and contract horizons. Simulation is used to quantify the credit risk exposure.

As guest editors of this special issue we would like to thank all who have contributed to its realization. First, we thank the editors of EJOR for their willingness to reserve room for the EURO working group on financial modelling and for their advice to the guest editors. Furthermore, we thank all the authors who have submitted their manuscripts. A special thank goes to the large number of anonymous referees. Their work has substantially contributed to the quality of the manuscripts and, consequently, to the quality of this issue. Finally, we thank our secretaries for their assistance in the editorial process. 